\author{
Fariba Yarandi ${ }^{1}$, Farahnaz Sadat \\ Ahmadi $^{2 *}$, Shamin Alaghi ${ }^{2}$, Azizeh \\ Ghaseminejad $^{1}$ and Soleiman Abbasi ${ }^{3}$ \\ ${ }^{1}$ Department of Obstetrics and Gynecology, General \\ Women Hospital, Tehran University of Medical \\ Sciences, Tehran, Iran \\ ${ }^{2}$ Department of Obstetrics and Gynecology, shohada \\ Hospital, Golestan University of Medical Sciences, \\ Golestan, Iran \\ ${ }^{3}$ Health Committee of the Islamic Parliament of Iran, \\ Tehran, Iran \\ Dates: Received: 01 August, 2015; Accepted: 10 \\ September, 2015; Published: 12 September, 2015 \\ *Corresponding author: Farahnaz Sadat Ahmadi, \\ Department of Obstetrics and Gynecology, Shohada \\ Hospital, Golestan University of Medical Sciences, \\ Golestan, Iran, Tel: 09111792944; Fax: 0098-17 \\ 33586039; E-mail: fsahmady@yahoo.com \\ www.peertechz.com
}

ISSN: 2455-5282

Keywords: Fallopian tube torsion; Acute abdomen Salpingectomy; Third trimester Pregnancy

\author{
Case Report
}

\section{Isolated Fallopian Tube Torsion after Eating Origan and Fennel during Pregnancy}

\section{Abstract}

Background: Isolated fallopian tube torsion is very rare during pregnancy . The reporter's show that the right fallopian tube torsion is most common. Most of the cases have presented in the third trimester. The diagnosis of this torsion is very difficult. There are not pathognomonic symptoms; clinical, imaging, or laboratory findings. The diagnosis is proved during the operation.

Case presentation: A 29 years old G4P3L2D1RIII, 33weeks pregnant woman presented with acute colicky abdominal pain with 4 episodes of vomiting \&nausea. The sonogram revealed a $2 \mathrm{~cm}$ exophytic mass of left ovary probably is haemorrhagic cyst.

A laparotomy was performed. The ovaries were normal and there was the isolated torsion in left tube with congestion and necrose. Cesarean section and salpingectomy was performed.

Conclusion: Although isolated twisted fallopian tube during pregnancy is very rare, we should Pay attention in the differential diagnosis of acute abdomen in pregnancy for this case. Early surgical intervention will decrease obstetric morbidity and may help us to preserve the fallopian tube.
\end{abstract}

\section{Introduction}

Isolated fallopian tube torsion is a rare event for abdominal pain during pregnancy [1]. First, it was described in 1890 [2]. The incidence is $1 / 1,5$ million women [3] only $12 \%$ of these cases are found during this pregnancy [4]. The right fallopian tube is most commonly affected about $90 \%$ and the Left fallopian tube is rare. preoperative correct diagnosis is rarely made.The condition is frequently misdiagnosed with torsion of the ovary, acute appendicitis, urolithiasis ,ectopic pregnancy, diverticulitis, degenerated leiomyoma, salpingitis, tuboovarian abscess, ruptured ovarian cyst, pelvic inflammatory disease and inflammatory bowel diseases $[5,6]$.

Accurate and immediate diagnosis can lead to early intervention in order to avoid complications and even save the fallopian tube organ [6].

\section{Case Report}

A 29 years old female G4P3L2D1RIII 33weeks pregnant woman was visited in obstetric clinic in Shohada hospital in Gonbad in Iran at 10 in the morning while complaining of colicky abdominal pain, with maximum intensity in left iliac fossa. The Pain was suddenly begun 2 hours ago. At first it was mild to moderate which progressively became severe. It was associated with nausea, and vomiting, no bowel or urinary symptoms. The patient said that she was eating origan and fennel for a cure for common cold without any consulting with doctors last night. Patient>s past history included hepatitis B and appendectomy, with no vaginal bleeding or other symptom.

On physical examination, there were focal tenderness and rebound tenderness in the left lower quadrant. As other exams were normal. Sonography examination showed a normal uterus and a normal right ovary. A $2 \mathrm{~cm}$ exophytic mass of left ovary probably haemorrhagic cyst. Obstetric sonography and fetal evaluation were normal. Total lab tests were normal. The report of surgery consult was expectant management. At the first day in the hospital, she was NPO. Second day she was not vomiting.

Nausea and abdominal tenderness was much reduced. The patient had appetite and had liquids.

The vital signs of patient were normal. With the surgical consultation, the patient was treated for diverticulitis. On the third day of hospitalization, the laparatomy was done in spite of mild contractions. The cesarean was completed. Adnexa on the right was completely normal. Similarly the left ovary was normal. While the left fallopian tube was found at the site of injection Ampula to Fimbriae torsion. According to fourth cesarean of the patient, salpingectomy was performed (Figures 1-4).

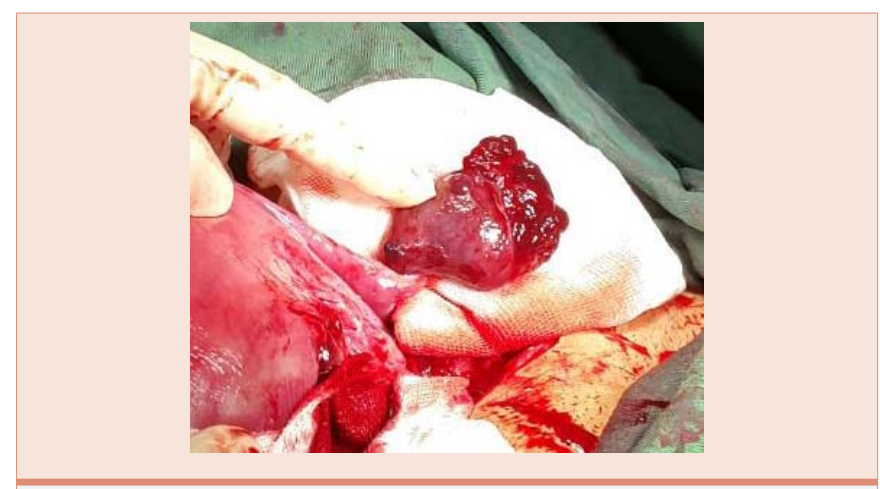

Figure 1: Isolated fallopian tube torsion. 


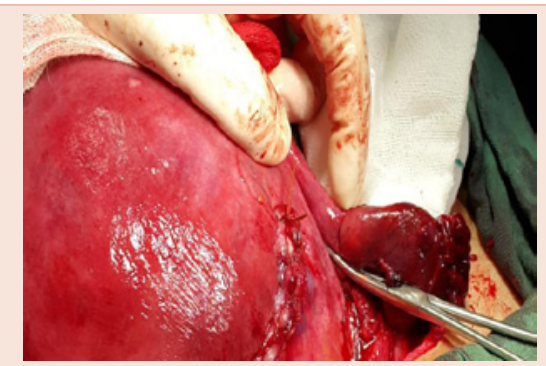

Figure 2: Location of twisted fallopian tube.

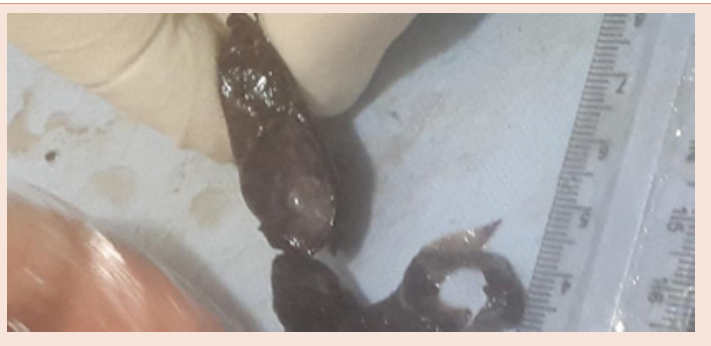

Figure 3: Paratubal cyst in fimbriae.

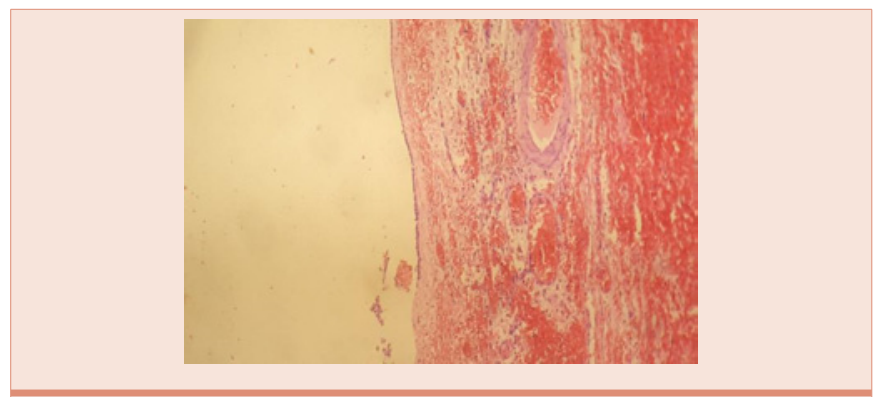

Figure 4: Hemorrhage in twisted fallopian tube.

Histopathologic examination showed congestion, hemorrhage and necrosis in the wall of the fallopian tube and Para tubal cyst was twisted in fallopian tube.

\section{Discussion}

Isolated tubal torsion is a rare gynecologic cause of acute abdominal pain, with a reported incidence of $1: 1,500,000$ women [3] only $12 \%$ of these causes occur during pregnancy [1,2].

Since there are no specific symptoms or physical signs for diagnosis of tubal torsion, ultrasound findings may be nonspecific and laboratory parameters maybe normal. About $90 \%$ of the times diagnosis is made during in the surgical exploration [4,7].

Presenting symptom is pain which begins sudden and cramp like in lower abdomen or pelvis but. The body temperature, erythrocyte sedimentation rate and white blood cell count may be normal or slightly elevated [15].

Examination may reveal focal tendernes and rebound tenderness in lower abdomen, tense adnexa associated with cervical tenderness [6].
The etiologies of twisted fallopian tube in this case were Para tubal cyst, pregnancy and may be eating origan and fennel. Other etiologies have been proposed including: anatomic abnormalities [tubal abnormalities, long mesosalpinx, hydrosalpinx, hematosalpinx], physiological abnormalities (hypermotility of the tube or abnormal peristalsis, tubal spasm from drugs), hemodynamic abnormalities (venous congestion in the mesosalpinx), Sellheim theory (sudden body position changes), trauma or disease (pelvic inflammatory disease, tubal ligation) and gravid uterus [9-16].

In cases of tubal torsion with an ischaemic event, the shorter the time from diagnosis to intervention plays an important role to prevent tubal necrosis and gangrenous changes. in the differential diagnosis of acute pain abdomen during pregnancy which can change the management, instead of salphingectomy a detorsion of the tube can be performed leading to fertility preservation in these young women [9].

But the prime question to be answered is when faced with a dilemmatic condition as in the present case. Pregnant woman in the 33th week of gestation and repeat IV and suggest the surgeon expectant management for diverticulitis when the concern is to salvage the present fetus and not all ovarian cysts in pregnancy or a red degeneration of fibroid need an exploration.

The situation with regard to the particular sensitivity of the patient's family circumstances (repeated IV, pregnancy age of 33 weeks and Hepatitis B) and infant. Especially when there are different opinions in the treatment the courage in decision-making is very important. In our case at the same time the baby was delivered by caesarean. Also salpingectomy was done. The new born received a course of betamethasone and didn't require NICU.

But the important question remained unanswered for us whether eating origan and fennel were associated with this process?

\section{Conclusions}

Although twisting or torsion of fallopian tube during pregnancy is Rare, it should be included in the differential diagnosis of acute abdomen in pregnancy. Early surgical intervention will decrease obstetric.

The important note on decision of proper treatment is, to consider all signs and symptoms of the patient. In this particular case, all the symptoms of the patient were significantly reduced or were disappeared. All the Paraclinic tests were normal but that did not justify me about persistence of the local tenderness. Despite opposition from the general surgeon, Laparotomy was done.

\section{References}

1. Adekanmi OA, Barrington JW, Edwaeds G, Farrell D (2000) Isolated torsion and hemorrhagic infarction of normal fallopian tube in an eleven yearold girl. BJOG 107; 1047-1048.

2. Sutton JB (1890) Remarks Salpingitis and some of its effects. Lancet 2: 12061209.

3. Hansen $\mathrm{OH}$ (1970) Isolated torsion of the Fallopian tube. Acta Obstet. Gynecol. Scand 49: 3-6.

4. Regad J (1993) Etude Anatomo-Pathologique de la torsion des trompets uterines. Gynecol Obstet 27: 519-535. 
5. Maynard SR, Peipert JF, Brody JM (1996) Tubal torsion appearing as acute pelvic inflammatory disease. J Am Assoc Gynecol Laparosc 3: 431-433.

6. ORIGONI M, CAVORETTO P, CONTI E, FERRARI A (2009) Isolated tuba torsion in pregnancy. Eur J Obstet Gynecol Reprod Biol 146: 116-120.

7. Isçi H, Güdücü N, Gönenç G, Basgul AY (2011) Isolated tubal torsion in pregnancy-a rare case. Clin Exp Obstet Gynecol 38: 272-273.

8. ten Cate A, Han S, Vliegen AS, Lewi L, Verhaeghe J, et al. (2011) Conservative surgery for left-sided isolated tubaltorsion in pregnancy. JBRBTR 94: 212-213.

9. Dueholm M, Praest J (1987) Isolated torsion of the normal fallopian tube. Acta Obstet Gynecol Scand 66: 89-90.

10. Chambers JT, Thiagarajah S, Kitchin JD III (1979) Torsion of the normal fallopian tube in pregnancy. Obstet Gynecol 54: 487-489.

11. Isager-Sally $L$, Weber $T$ (1985) Torsion of the fallopian tube during pregnancy. Acta Obstet Gynecol Scand 64: 349-351.
12. McKenna PJ, Gerbert KH (1989) Isolated torsion of the uterine tube in pregnancy. A case report. J Reprod Med 34: 187-188.

13. Yalcin OT, Hassa H, Zeytinoglu S, Isiksoy S (1997) Isolated torsion of fallopian tube during pregnancy; report of two cases. Eur J Obstet Gynecol Reprod Biol 74: 179-182.

14. Sorem KA, Bengtson JM, Walsh B (1991) Isolated fallopian tube torsion presenting in labor. A case report. J Reprod Med 36: 763-764.

15. Milki A, Jacobson DH (1998) Isolated torsion of the fallopian tube. A case report. J Reprod Med 43: 836-838.

16. Provost RW (1972) Torsion of the normal fallopian tube.Obstet Gynecol 39: 80-82.

17. Elchalal U, Caspi B, Schachter M, Borenstein R (1993) Isolated tubal torsion: clinical and ultrasonographic correlation.J Ultrasound Med 12: 115-117.

Copyright: (c) 2015 Yarandi $F$, et al. This is an open-access article distributed under the terms of the Creative Commons Attribution License, which permits unrestricted use, distribution, and reproduction in any medium, provided the original author and source are credited. 